\section{A pilot clinical study of low-intensity transcranial focused ultrasound in Alzheimer's disease}

\author{
Hyeonseok Jeong ${ }^{1,2,3 *}$, Jooyeon Jamie $\mathrm{Im}^{1,2 *}$, Jong-Sik Park², Seung-Hee $\mathrm{Na}^{2}$, \\ Wonhye Lee ${ }^{4}$, Seung-Schik Yoo ${ }^{4}$, In-Uk Song ${ }^{2}$, Yong-An Chung ${ }^{1,3}$ \\ Departments of ${ }^{1}$ Nuclear Medicine, ${ }^{2}$ Neurology, and ${ }^{3}$ Radiology, Incheon St. Mary's Hospital, \\ College of Medicine, The Catholic University of Korea, Seoul, Korea; ${ }^{4}$ Department of \\ Radiology, Brigham and Women's Hospital, Harvard Medical School, Boston, MA, USA
}

Purpose: Increasing attention has been paid to low-intensity transcranial focused ultrasound (tFUS) for its potential therapeutic effects in Alzheimer's disease (AD). While preclinical studies have shown promising therapeutic effects of low-intensity tFUS in AD models, its efficacy and safety remain unclear in humans. In this pilot study, we investigated the effects of low-intensity tFUS on blood-brain barrier opening, the regional cerebral metabolic rate of glucose (rCMRglu), and cognition in patients with AD.

Methods: After receiving institutional review board approval, four patients with AD received tFUS to the hippocampus immediately after an intravenous injection of a microbubble ultrasound contrast agent. Sonication was delivered at low-intensity, at a pressure level below the threshold for blood-brain barrier opening. Patients underwent brain magnetic resonance imaging, ${ }^{18} \mathrm{~F}$-fluoro-2-deoxyglucose positron emission tomography, and neuropsychological assessments before and after the tFUS procedure. A whole-brain voxel-wise paired t test was conducted to compare rCMRglu before and after tFUS.

Results: The sonication, as anticipated, did not show evidence of active blood-brain barrier opening on $\mathrm{T1}$ dynamic contrast-enhanced magnetic resonance imaging. rCMRglu in the superior frontal gyrus $(P<0.001)$, middle cingulate gyrus $(P<0.001)$, and fusiform gyrus increased after tFUS $(P=0.001)$. Patients demonstrated mild improvement in measures of memory, executive, and global cognitive function following tFUS. No adverse events were reported.

Conclusion: These results suggest that hippocampal sonication with low-intensity tFUS may have beneficial effects on cerebral glucose metabolism and cognitive function in patients with AD. Further larger studies are needed to confirm the therapeutic efficacy of tFUS in AD.

Keywords: Low-intensity transcranial focused ultrasound; Alzheimer's disease; Regional cerebral metabolic rate of glucose; Cognitive function

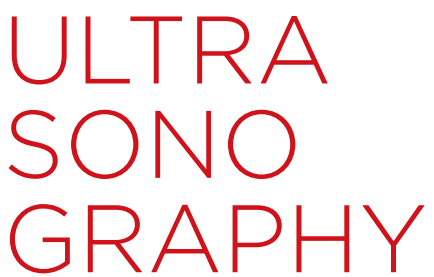

ORIGINAL ARTICLE

https://doi.org/10.14366/usg.20138 pISSN: 2288-5919 • elSSN: 2288-5943 Ultrasonography 2021;40:512-519

Received: September 4, 2020 Revised: December 23, 2020 Accepted: January 16, 2021

Correspondence to: In-Uk Song, MD, PhD, Department of Neurology, Incheon St. Mary's Hospital, College of Medicine, The Catholic University of Korea, 56 Dongsu-ro, Bupyeong-gu, Incheon 21431, Korea

Tel. +82-32-280-5010

Fax. +82-32-280-5244

E-mail: siuy@catholic.ac.kr

Yong-An Chung, MD, PhD, Department of Nuclear Medicine, Incheon St. Mary's Hospital, College of Medicine, The Catholic University of Korea, 56 Dongsu-ro, Bupyeong-gu, Incheon 21431, Korea

Tel. +82-32-280-5242

Fax. +82-32-280-5244

E-mail: yongan@catholic.ac.kr

*These authors contributed equally to this work.

This is an Open Access article distributed under the terms of the Creative Commons Attribution NonCommercial License (http://creativecommons.org/ licenses/by-nc/4.0/) which permits unrestricted noncommercial use, distribution, and reproduction in any medium, provided the original work is properly cited.

Copyright @ 2021 Korean Society of Ultrasound in Medicine (KSUM)

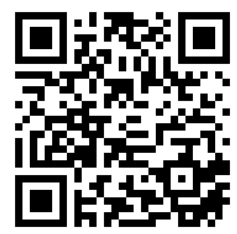

How to cite this article:

Jeong H, Im JJ, Park JS, Na SH, Lee W, Yoo SS, et, al. A pilot clinical study of low-intensity transcranial focused ultrasound in Alzheimer's disease. Ultrasonography. 2021 0ct;40(4):512519. 


\section{Introduction}

Alzheimer's disease (AD) is the most common neurodegenerative disorder, and is characterized by progressive cognitive decline and neuropathologically by the presence of neuritic plaques and neurofibrillary tangles [1]. Plaques and tangles, which primarily consist of amyloid-beta and hyperphosphorylated tau, respectively, are highly concentrated in brain regions that are important for memory and cognition such as the hippocampus and cerebral cortex [2]. Despite the increasing prevalence and debilitating consequences of $A D$, current treatment methods have limited efficacy in preventing, slowing, or stopping the progression of the disease. It is therefore a high priority to find effective treatment strategies for AD.

Ultrasound has been shown to produce diverse biological effects via thermal/non-thermal, mechanical, or electrophysiological interactions with biological tissues, which may yield impactful therapeutic outcomes [3]. The advent of focused ultrasound technology has enabled the application of acoustic energy to highly specific intracranial areas, including deep brain regions, with spatial specificity on the order of millimeter to submillimeter resolution [4]. Low-intensity transcranial focused ultrasound (tFUS) is an emerging brain stimulation method, which could excite or inhibit neural activity in a reversible and non-invasive manner without raising tissue temperature [5]. In conjunction with intravenous injection of microbubble ultrasound contrast agents (MBs), the use of tFUS has also been investigated as a non-invasive means to transiently open the blood-brain barrier (BBB) in a region-specific fashion [6]. Although the exact mechanism requires further study, acoustic cavitation of MBs induced by tFUS is believed to amplify local pressure waves and thereby temporarily stretch the endothelial walls of the BBB around the targeted brain area [6].

tFUS-mediated BBB disruption has been utilized to enhance the delivery of exogenous therapeutics [7-9]. In a transgenic rodent model of AD, MB-tFUS-mediated delivery of antibodies against tau protein and amyloid across the BBB reduced tau and amyloid levels and had positive effects on memory performance [8]. Strikingly, in further investigations using an AD mouse model, BBB disruption alone, even without introducing exogenous therapeutic agents, led to a reduction in the plaque burden, promotion of neuronal plasticity, and improvement in spatial memory [10]. A similar tFUSBBB disruption procedure, without the introduction of exogenous drug molecules, targeting the hippocampus in normal adult mice exhibited positive generation/proliferation of neurons [11]. Building upon this compelling preclinical evidence, tFUS-mediated human $B B B$ disruption trials for $A D$ patients are ongoing, targeting the dorsolateral prefrontal cortex [4], anterior right frontal lobe [12], and memory circuits (i.e., the hippocampus/entorhinal cortex) [13]. Such studies have demonstrated that repetitive BBB disruption sessions can be safely delivered in AD patients, prompting further studies to examine group-wise changes in clinical symptoms and function over time.

The purpose of this study was to evaluate the safety and efficacy of low-intensity tFUS under the threshold for BBB disruption in patients with AD. To do so, we applied low-intensity tFUS to the unilateral hippocampal area of patients with $A D$ and evaluated the safety and presence of BBB disruption using magnetic resonance imaging (MRI). In addition, we assessed changes in the regional cerebral metabolic rate of glucose (rCMRglu) using ${ }^{18} \mathrm{~F}$-fluoro2-deoxyglucose positron emission tomography (FDG-PET) and cognitive function after tFUS.

\section{Materials and Methods}

\section{Compliance with Ethical Standards}

This single-center prospective study was approved by the Institutional Review Board of Incheon St. Mary's Hospital in Korea (OC18DESI0130). All participants and their legal guardians provided written informed consent.

\section{Study Population}

$A D$ patients aged between 65 and 85 years were recruited from the neurology outpatient clinic between August 2019 and November 2019. The clinical diagnosis of AD was based on the Diagnostic and Statistical Manual of Mental Disorders-IV criteria [14] and the National Institute of Neurological and Communicative Disorders and Stroke and the Alzheimer's Disease and Related Disorders Association criteria [15]. The inclusion criteria also included a Clinical Dementia Rating (CDR) score between 1 and 3 and a MiniMental State Examination (MMSE) score below 20. The exclusion criteria were the following: radiological findings on MRI, epilepsy, stroke, mixed or vascular dementia, other neurological or psychiatric disorders, and contraindications to MRI, MRI contrast agents, or MBs. The overall process of the study is summarized in Fig. 1.

\section{Clinical Assessments}

The screening tests included medical history-taking, physical and neurological examinations conducted by a neurologist, routine blood biochemistry and blood count, blood lipid profile, 12-lead electrocardiography (ECG), and a chest X-ray examination.

The patient's general cognitive status and severity of dementia were evaluated by MMSE, CDR, and the CDR-Sum of Boxes (CDRSOB). Moreover, a neuropsychological test battery was composed of subtests assessing attention (Digit Span Test: forward and backward), visuospatial function (Clock Drawing Test, Rey Complex 


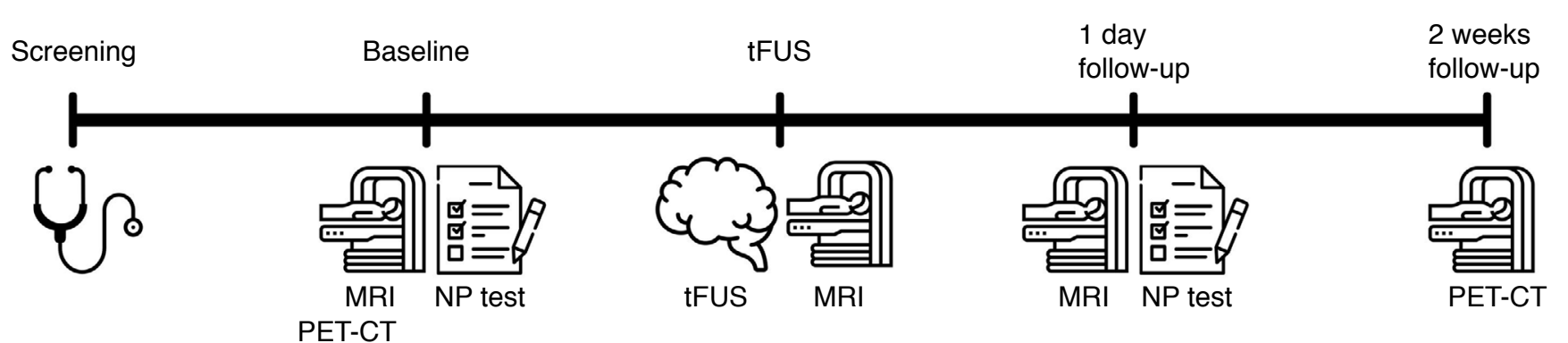

Fig. 1. Schematic of the overall study process. Before the tFUS procedure, baseline neuropsychological tests and MRI, CT, and PET imaging of the head were conducted. Low-intensity tFUS was administered to the right hippocampus with microbubble ultrasound contrast agents. Immediately after the procedure, patients underwent brain MRI to evaluate the presence of blood-brain barrier opening. One day after the tFUS procedure, patients underwent follow-up MRI and neuropsychological tests. Follow-up PET scans were performed approximately 2 weeks after the tFUS procedure. tFUS, transcranial focused ultrasound; MRI, magnetic resonance imaging; CT, computed tomography; PET, positron emission tomography; NP, neuropsychological.

Figure Test: copy), memory (Seoul Verbal Learning Test-Elderly's version [SVLT-E]: immediate recall, delayed recall, and recognition), and executive function (short form of the Korean-Color Word Stroop Test: word reading and color reading, Controlled Oral Word Association Test: Animal and Phonemic, Contrasting Program, and Go/No-Go Test). The neuropsychological assessment was conducted by a licensed neuropsychologist blinded to the purpose of this study.

Each patient was admitted to the hospital 1 day before the planned tFUS procedure and remained hospitalized for approximately 24 hours after the procedure to monitor adverse events such as vital sign abnormalities, skin changes, microhemorrhages on susceptibilityweighted imaging (SWI), and lesions on fluid-attenuated inversion recovery (FLAIR) imaging. To monitor delayed adverse events, the patient was followed up through subsequent outpatient care.

\section{Baseline Imaging Acquisition}

Before the tFUS procedure, MRI and computed tomography (CT) scans were conducted for image guidance of sonication targeting $[16,17]$. Four adhesive fiducial markers (visible in both MRI/CT scans, PinPoint, Beekley Corp., Bristol, CT, USA) were placed on spatially distributed locations over the head. The spatial coordinates of the fiducial markers in MRI/CT data were used to register the individual's physical space to the multi-modal data's virtual space in order to align the tFUS focus to the targeted hippocampal area.

The brain MRI data was obtained using a 3-T clinical MR scanner (MAGNETOM Skyra, Siemens, Enlargen, Germany) with a 32-channel head coil. High-resolution T1-weighted anatomical MRI was acquired using the 3D magnetization-prepared rapid gradient-echo sequence (repetition time [TR], 2,000 ms; echo time [TE], $2.49 \mathrm{~ms}$; flip angle, $9^{\circ}$; field of view [FOV], $230 \times 230 \mathrm{~mm}^{2}$; matrix, $256 \times 256$ [phasexfrequency]; voxel size, $0.9 \times 0.9 \times 0.9 \mathrm{~mm}^{3}$; bandwidth, 180 $\mathrm{Hz} /$ pixel; 224 sagittal slices). In addition, FLAIR imaging (TR, 9,000 ms; TE, 81 ms; inversion time, 2,500; flip angle, 90; FOV, 224×224 $\mathrm{mm}^{2}$; matrix, $256 \times 320$ [phase $\times$ frequency]; voxel size, $0.7 \times 0.7 \times 4.0$ $\mathrm{mm}^{3}$; bandwidth, $289 \mathrm{~Hz} /$ pixel; 44 slices) and SWI (TR, $27 \mathrm{~ms}$; $\mathrm{TE}, 20 \mathrm{~ms}$; flip angle, $15^{\circ}$; FOV, $203 \times 224 \mathrm{~mm}^{2}$; matrix, $232 \times 256$ [phasexfrequency]; voxel size, $0.44 \times 0.44 \times 1.50 \mathrm{~mm}^{3}$; bandwidth, $120 \mathrm{~Hz} /$ pixel; 104 slices) were performed to detect anatomical abnormalities and the presence of hemorrhage.

Brain FDG-PET and CT scans were performed using a Discovery STE PET-CT scanner (GE Healthcare, Milwaukee, WI, USA). All participants were injected with 185-222 MBq of FDG intravenously and rested in a supine position with eyes closed in a quiet and dark room for 45 minutes. A total of 47 transaxial emission images were obtained (pixel size, $1.95 \times 1.95 \mathrm{~mm}$; slice thickness, $3.27 \mathrm{~mm}$; matrix, 128×128). CT images were also acquired (FOV, 250×250 $\mathrm{mm}^{2}$; matrix, 512×512; voxel size, $0.49 \times 0.49 \times 0.50 \mathrm{~mm}^{3}$ ). Standard filtering techniques and the ordered subset expectation maximization algorithm were applied to reconstruct the PET images. CT images were examined for the presence of large calcifications within the cranial cavity ( $>3 \mathrm{~mm}$, one-half of the wavelength of the ultrasound waves), which may perturb the propagation of acoustic waves; none were found.

\section{Low-Intensity tFUS}

We applied low-intensity tFUS to the right hippocampus for 3 minutes using an image-guided tFUS system (NS-US100, Neurosona Co., Ltd., Seoul, Korea). For multi-modal neuroimaging guidance, MRI and CT data were spatially co-registered using the maximization of normalized mutual information [18]. The subjectspecific coordinates of the hippocampus in the right hemisphere were identified as the tFUS target location. Based on CT data, the sonication path was planned to be perpendicular to the skull at the entry while avoiding the sinus and thick skull segments (> 10 mm) 
along the beam path. A compressible cryogel (Bluemtech, Wonju, Korea) was applied between the transducer and the scalp to provide acoustic coupling (Fig. 2A).

The spatial acoustic intensity profile generated by the tFUS transducer showed that the size of the tFUS focus at full-width at half-maximum intensity was $5.8 \mathrm{~mm}$ in diameter and $37.3 \mathrm{~mm}$ in length (Fig. 2B). The center of the maximum intensity area was 70 $\mathrm{mm}$ away from the exit plane of the transducer on the beam path. The procedure of mapping the acoustic intensity field has been described elsewhere [17].

With the patient comfortably seated on a recliner chair, the physical space of the head was registered to the space of the T1weighted images under optical tracking (NDI, Ontario, Canada)
$[16,17]$. Ultrasound hydrogel (Aquasonic, Parker Laboratories, Fairfield, NJ, USA) was applied around the entry point on the scalp. Intravenous catheterization was prepared prior to the sonication session. The operator aligned the acoustic focus to the right hippocampus (Fig. 2B) and administered MBs (Definity, Lantheus Medical Imaging Inc., North Billerica, MA, USA) intravenously at a dose of $10 \mu \mathrm{L} / \mathrm{kg}$ over a $\sim 1$-minute period. Immediately after MB injection, low-intensity tFUS was delivered immediately afterward to the hippocampal target using the following sonication parameters: a fundamental frequency of $250 \mathrm{kHz}$, and a tone-burst-duration of 20 ms with a pulse repetition frequency of $2 \mathrm{~Hz}$ (i.e., a duty cycle of $4 \%$ ). The acoustic intensity at the focal target had a spatial-peak pulseaverage acoustic intensity of $0.5-3 \mathrm{~W} / \mathrm{cm}^{2}$, resulting in a spatial-
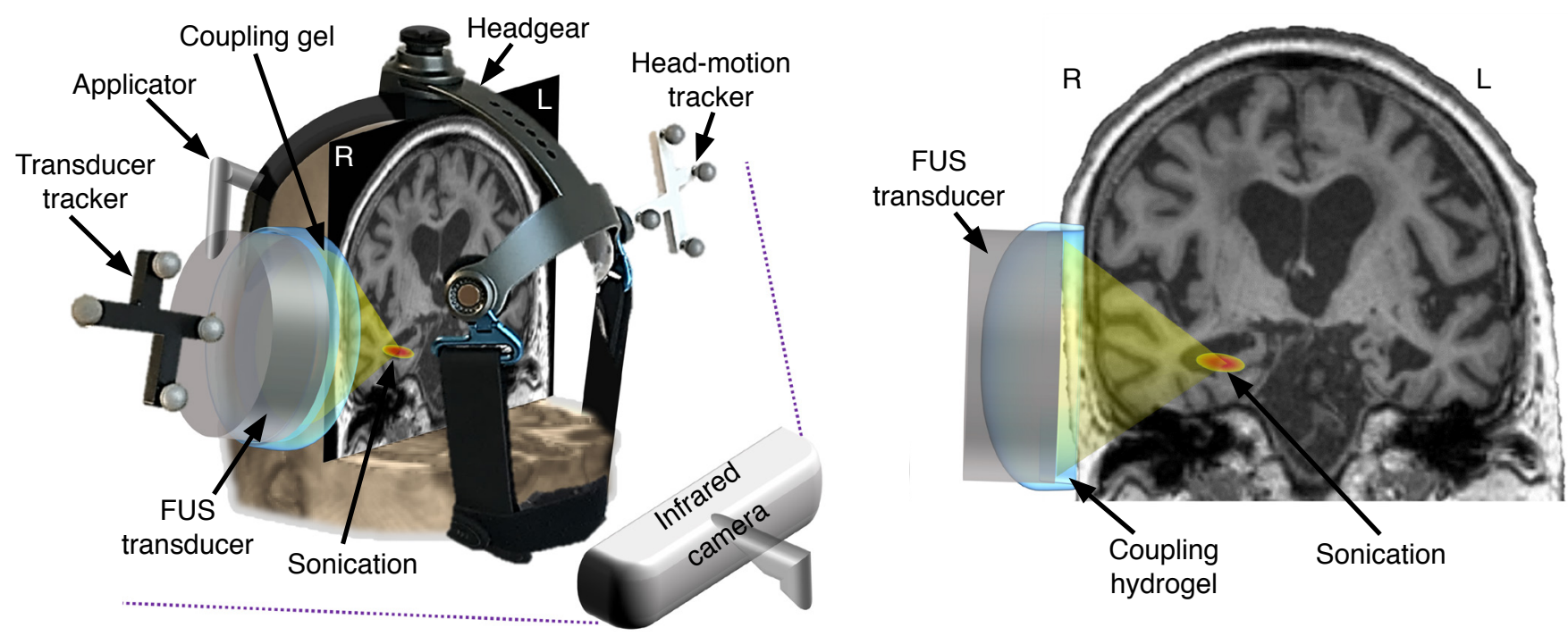

A
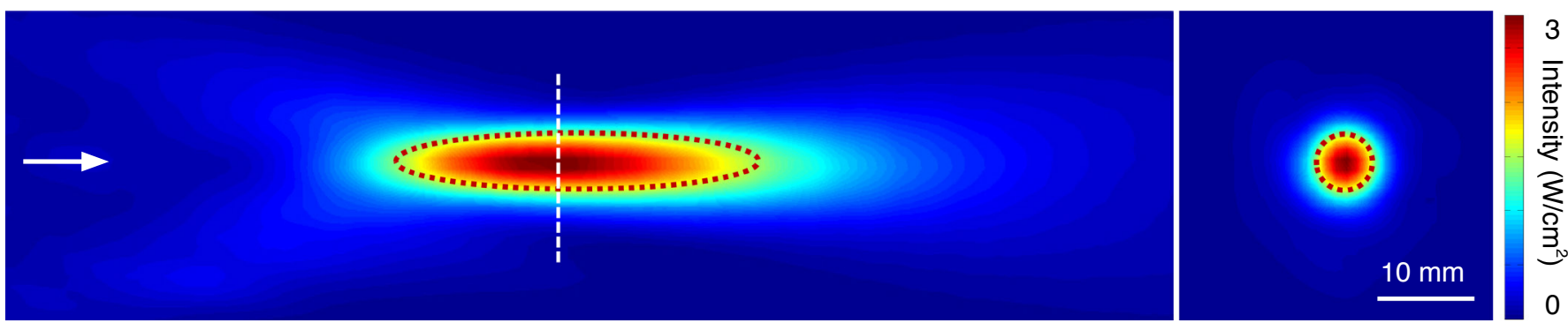

B

Fig. 2. Schematic of the experimental setup.

A. Illustration of transcranial focused ultrasound (tFUS) sonication of the planned target (right hippocampus) is shown. An optical tracker was attached to the ergonomic headgear and the tFUS transducer. The location of acoustic focus was navigated with respect to the head anatomy using a motion-tracking infrared camera. An illustration of the sonication path is overlaid on the T1-weighted anatomical image with the tFUS transducer positioned over the entry point on the scalp. A compressible hydrogel was placed between the transducer and the scalp for acoustic coupling. B. Characterization of the acoustic intensity profile in the longitudinal plane along the beam path and transverse plane (at the location of the white dotted line) perpendicular to the beam is shown. The white arrow represents the direction of sonication. The full-width at half-maximum intensity profile is indicated by the dotted orange ellipse and circle. Scale bar=10 mm. L, left; $R$, right. 
peak temporal-average acoustic intensity of $0.02-0.12 \mathrm{~W} / \mathrm{cm}^{2}$.

\section{Follow-up Imaging Acquisition}

After the tFUS procedure, patients underwent additional MRI. The lead time between the end of the FUS procedure and the start of the MRI scan was approximately 30 minutes. After the SWI and FLAIR scans, T1-weighted images were acquired with two different flip angles of $2^{\circ}$ and $14^{\circ}$ (TR, $4.42 \mathrm{~ms} ; \mathrm{TE}, 2.01 \mathrm{~ms} ;$ FOV, $224 \times 224$ $\mathrm{mm}^{2}$; matrix, $224 \times 224$ [phasexfrequency]; voxel size, $1.0 \times 1.0 \times 3.0$ $\mathrm{mm}^{3}$; bandwidth, $450 \mathrm{~Hz} /$ pixel; 52 slices). For T1 dynamic contrast-enhanced (DCE)-MRI, a 3D gradient-echo sequence (3D CAIPIRINHA-controlled aliasing in parallel imaging results in higher acceleration) was used at TR, $3.32 \mathrm{~ms}$; TE, $1.12 \mathrm{~ms}$; flip angle, $9^{\circ}$, with 24 reference lines for both phase and partition encoding using an acceleration factor of $2 \times 2$ (phasexpartition) with reordering shift 1, FOV, $224 \times 224 \mathrm{~mm}^{2}$; matrix, $224 \times 224$ (phasexfrequency); voxel size, $1.0 \times 1.0 \times 4.0 \mathrm{~mm}^{3}$ (44 slices). In total, 48 volumes were acquired in a total scan duration of 10 minutes and 11 seconds. Intravenous injection of gadobutrol (Gadovist, Bayer Healthcare, Wayne, NJ, USA) was given at a dose of $0.1 \mathrm{~mL} / \mathrm{kg}$ before the DCEMRI sequence, using an automated injector with a flow rate of 2.5 $\mathrm{mL} / \mathrm{s}$, followed by a $25-\mathrm{mL}$ saline flush with a flow rate of $2.0 \mathrm{~mL} / \mathrm{s}$. The MRI scans were repeated 1 day after the tFUS procedure using the same protocol. In addition, follow-up FDG-PET scans were performed approximately 2 weeks after the tFUS procedure.

\section{Image Analysis}

For each patient, the presence of BBB opening was evaluated by a visual inspection of contrast enhancement and independent component analysis (ICA) of DCE-MRI data. For the ICA analysis, all volumes of each patient were realigned using Statistical Parametric Mapping 12 (SPM; https://www.fil.ion.ucl.ac.uk/spm). The images were then skull-stripped and merged to obtain time-series of DCEMRI images using FMRIB Software Library (https://fsl.fmrib.ox.ac.uk/ fsl/fslwiki). The Group ICA of fMRI Toolbox (GIFT; https://trendscenter. org/software/gift) was used to decompose the preprocessed data from each patient into 20 independent components (ICS) based on the Infomax algorithm [19]. The stability of the estimated ICs was tested by repeating the ICA algorithm 20 times using the ICASSO toolbox [20], implemented in GIFT. Components that demonstrated an initial signal increase after gadobutrol injection followed by sustained elevation were selected and visually inspected.

All PET images were spatially normalized to the standard PET template, resliced with a voxel size of $2 \times 2 \times 2 \mathrm{~mm}^{3}$, and smoothed with an 8-mm full-width at half-maximum isotropic Gaussian kernel using SPM. Relative tracer activity at each voxel was estimated as the ratio to mean cerebellar activity using proportional scaling.
For voxel-wise analysis, the paired t test was performed to assess changes in rCMRglu before and after sonication. The voxel-wise significance threshold was set at $\mathrm{P}<0.005$ (uncorrected) with a minimum cluster size of 50 contiguous voxels.

\section{Results}

Four patients (mean age \pm standard deviation, 78.8 \pm 3.3 years; 3 women) with probable $A D$ were enrolled in this study. No patients showed any abnormalities on blood biochemistry and count, ECG, or the chest X-ray examination before the tFUS procedure. The visual inspection and ICA analysis of the DCE-MRI data revealed no evidence of contrast enhancement related to BBB disruption. No adverse events were observed after the procedure or in outpatient follow-up visits for up to 1 year.

The results of the neuropsychological tests before and after tFUS are summarized in Table 1. Overall, mild improvement was observed in global cognitive function (MMSE), executive function (Contrasting Program, Go/No-Go Test), and memory (SLVT-E: immediate recall and recognition).

The PET analysis revealed a significant increase in rCMRglu

Table 1. Neuropsychological tests before and after transcranial focused ultrasound

\begin{tabular}{lcccc}
\hline & $\begin{array}{c}\text { Patient 1 } \\
\text { (before/ } \\
\text { after) }\end{array}$ & $\begin{array}{c}\text { Patient 2 } \\
\text { (before/ } \\
\text { after) }\end{array}$ & $\begin{array}{c}\text { Patient 3 } \\
\text { (before/ } \\
\text { after) }\end{array}$ & $\begin{array}{c}\text { Patient 4 } \\
\text { (before/ } \\
\text { after) }\end{array}$ \\
\hline MMSE & $10 / 11$ & $13 / 15$ & $7 / 7$ & $6 / 9$ \\
CDR & $2 / 2$ & $1 / 1$ & $2 / 2$ & $2 / 2$ \\
CDR-SOB & $12 / 12$ & $7 / 7$ & $12 / 12$ & $12 / 12$ \\
Digit Span - Forward & $3 / 3$ & $5 / 6$ & $4 / 4$ & $5 / 5$ \\
Digit Span - Backward & $0 / 0$ & $2 / 2$ & $0 / 0$ & $2 / 2$ \\
Clock Drawing & $1 / 1$ & $1 / 1$ & $0 / 0$ & $0 / 0$ \\
RCFT - Copy Score & $1 / 1.5$ & $\mathrm{n} / \mathrm{a}$ & $\mathrm{n} / \mathrm{a}$ & $1.5 / 0.5$ \\
SVLT-E - Immediate Recall & $2 / 3$ & $0 / 1$ & $0 / 2$ & $4 / 4$ \\
SVLT-E - Delayed Recall & $0 / 0$ & $0 / 0$ & $0 / 0$ & $0 / 0$ \\
SVLT-E - Recognition & $11 / 15$ & $11 / 12$ & $12 / 12$ & $12 / 12$ \\
K-CWST - Word Reading & $\mathrm{n} / \mathrm{a}$ & $112 / 109$ & $\mathrm{n} / \mathrm{a}$ & $51 / 24$ \\
K-CWST - Color Reading & $\mathrm{n} / \mathrm{a}$ & $0 / 0$ & $\mathrm{n} / \mathrm{a}$ & $11 / 7$ \\
COWAT - Animal & $3 / 5$ & $2 / 0$ & $0 / 0$ & $2 / 2$ \\
COWAT - Phonemic & $0 / 0$ & $0 / 0$ & $0 / 0$ & $0 / 0$ \\
Contrasting Program & $0 / 3$ & $10 / 19$ & $0 / 0$ & $3 / 15$ \\
Go/No-Go & $0 / 5$ & $0 / 9$ & $0 / 0$ & $8 / 7$ \\
\hline MMSE, Mni-Mental State Exam & & & & \\
\hline
\end{tabular}

MMSE, Mini-Mental State Examination; CDR, Clinical Dementia Rating; CDR-SOB, Clinical Dementia Rating-Sum of Boxes; RCFT, Rey Complex Figure Test; n/a, not assessed because patients were either illiterate or uncooperative; SVLT-E, Seoul Verbal Learning Test-Elderly's version; K-CWST, Korean-Color Word Stroop Test; COWAT, Controlled Oral Word Association Test. 
following low-intensity tFUS in the right superior frontal gyrus (6.6\%, $\mathrm{P}<0.001)$, right middle cingulate gyrus $(5.5 \%, \mathrm{P}<0.001)$, and left fusiform gyrus (5.4\%, $P=0.001$ ) (Table 2, Fig. 3). No significant decrease in rCMRglu was observed following tFUS.

\section{Discussion}

We applied low-intensity tFUS to the unilateral hippocampal area in $A D$ patients who received intravenous ultrasound contrast agent. As expected, since we used an ultrasound pressure level under the threshold for BBB opening, we found that tFUS did not result in BBB opening. Even without BBB opening, tFUS increased rCMRglu in the frontal, cingulate, and temporal lobes, and improved general

Table 2. Changes of brain glucose metabolism after transcranial focused ultrasound

\begin{tabular}{|c|c|c|c|c|}
\hline Region & $\mathrm{t}$ & P-value & $\begin{array}{c}\text { Coordinates }^{a)} \\
(x, y, z)\end{array}$ & $\begin{array}{c}\text { Cluster size } \\
\text { (voxels) }\end{array}$ \\
\hline \multicolumn{5}{|l|}{$\begin{array}{l}\text { Increased regional cerebral } \\
\text { metabolic rate of glucose }\end{array}$} \\
\hline Right superior frontal gyrus & 15.21 & $<0.001$ & $16,4,58$ & 53 \\
\hline Right middle cingulate gyrus & 14.62 & $<0.001$ & $14,-16,46$ & 57 \\
\hline Left fusiform gyrus & 10.37 & 0.001 & $-36,-40,-22$ & 53 \\
\hline \multicolumn{5}{|l|}{$\begin{array}{l}\text { Decreased regional cerebral } \\
\text { metabolic rate of glucose }\end{array}$} \\
\hline None & - & - & - & - \\
\hline
\end{tabular}

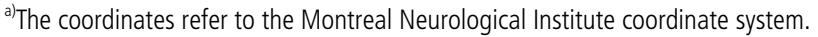

cognitive function, executive function, and memory in patients with AD. Moreover, no acute or delayed adverse events were observed following tFUS. To the best of our knowledge, our study is the first report of tFUS-induced effects on cerebral glucose metabolism and cognition in patients with $A D$.

In our study, DCE-MRI following the tFUS session showed no evidence of $\mathrm{BBB}$ disruption. As pressure and the associated mechanical index (MI) are important indicators for achieving BBB disruption [6], we conjecture that the acoustic pressure level used in this study (in situ pressure of $\sim 135 \mathrm{kPa}$ and $\mathrm{Ml}$ of 0.27 ), which was under the threshold for creating stable cavitation of MBs, was not high enough to cause BBB disruption. This conjecture is consistent with a previous investigation involving sheep in which localized enhancement of BBB permeability occurred in regions subjected to acoustic pressure levels greater than $480 \mathrm{kPa}$ (MI, 0.96) after intravenous injection of the same type of MBs [21]. It is also in agreement with a study by Hynynen and colleagues [22], which reported that an in situ pressure of $200 \mathrm{kPa}$ (MI of $>0.4$ ) was needed to induce $\mathrm{BBB}$ disruption when $0.05 \mathrm{~mL} / \mathrm{kg}$ dose of MBs (Optison, GE Healthcare, Princeton, NJ, USA) was used in rabbits at a similar tFUS frequency (i.e., $260 \mathrm{kHz}$ ) and pulsing scheme (10 ms tone-burst duration, $1 \mathrm{~Hz}$ sonication for $20 \mathrm{~s}$ ). As successful BBB disruption depends on many factors, such as the type of MBs, sonication parameters, and transducer configuration, and given that excessive disruption may cause deleterious effects such as microhemorrhage [6], cautionary countermeasures such as acoustic

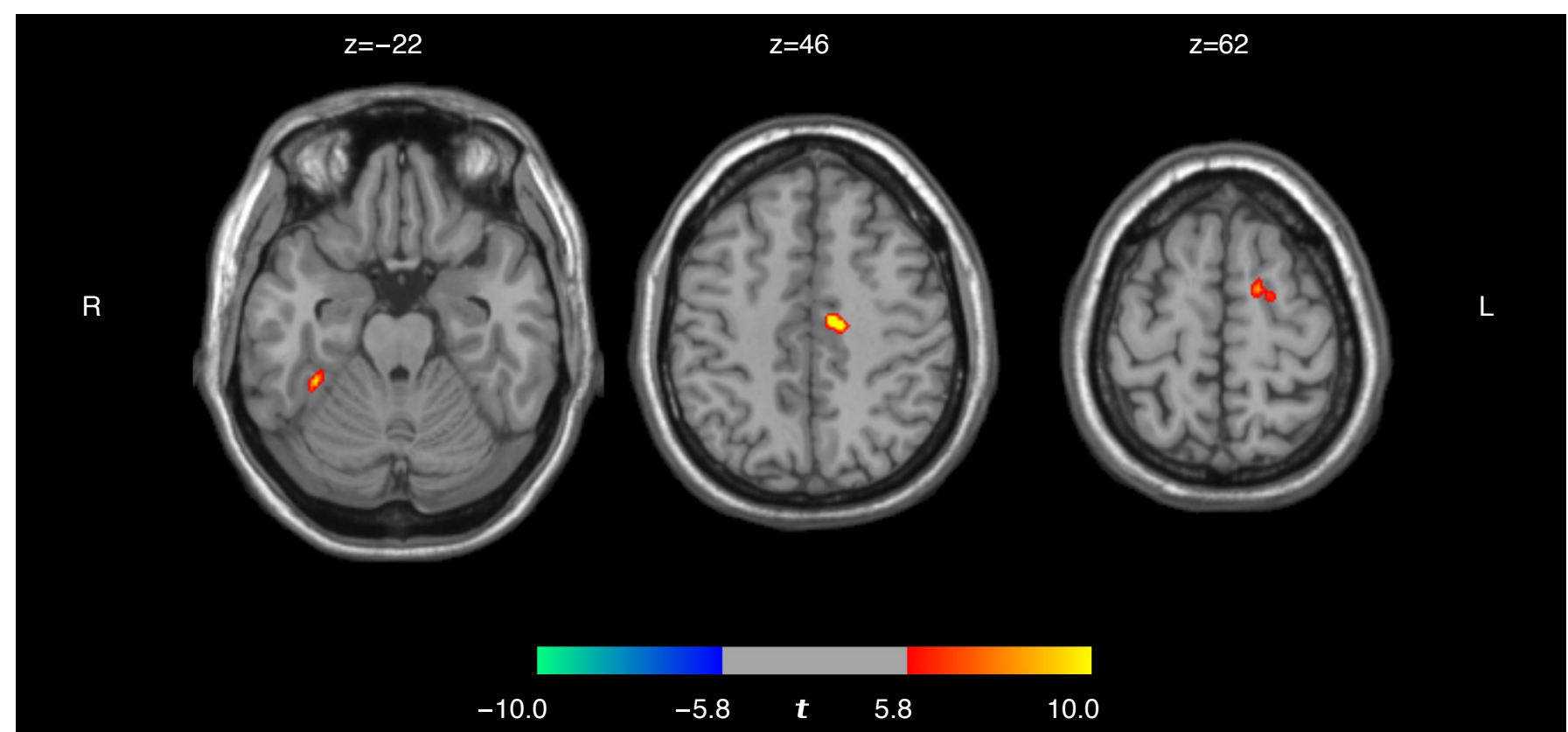

Fig. 3. Changes in regional cerebral metabolic rate of glucose (rCMRglu) following transcranial focused ultrasound. Increases (red-yellow) or decreases (blue-green) of rCMRglu at follow-up compared with baseline are presented. The numbers above the brain slices indicate $z$ coordinates in the Montreal Neurological Institute space. The color bar represents voxel-level t-values. L, left; R, right. 
emission detection of undesirable inertial cavitation [23] are needed to promote the safety of the procedure.

We showed that hippocampal sonication with low-intensity tFUS improved rCMRglu in the temporal, cingulate, and frontal cortices. Moreover, mild improvement was noted in measures of global cognitive function, memory, and executive function. Although previous studies reported tFUS-induced BBB opening and $\beta$-amyloid plaque reduction in patients with $A D$, improvements in cognition or brain function were not observed $[4,13]$. Previous FDG-PET studies reported that AD patients show reduced rCMRglu in the parietal-temporal and frontal cortices in advanced stages [24]. Our results suggest that low-intensity tFUS may have exerted potential therapeutic effects on impaired brain function, which is related to cognitive decline. Although it is not clear how the effects of hippocampal sonication extended beyond the close proximity of the target site, we suspect that improvement in frontal lobe function may be related to potential functional modulation of the hippocampal-prefrontal cortex (H-PF) pathway by the application of tFUS. The H-PF pathway is a major monosynaptic input to the prefrontal cortex originating in the hippocampal formation [25]. The literature based on animal and human experiments supports the theory that the pathway is involved in fundamental cognitive processes including executive function and memory and contextually-dependent emotional regulation [26]. Although the exact mechanism behind tFUS-mediated neuromodulation remains to be elucidated, it is plausible that FUS application to the hippocampal area, even without changing BBB permeability, may increase activity in the hippocampal area and modulate neural activity of the H-PF pathway, resulting in the observed improvement in memory.

Some limitations of this study need to be addressed. First, this study was conducted among a small number of patients. For this reason, we did not perform a quantitative analysis of the neuropsychological data. Another potential limitation is that we only included $A D$ patients with an overall symptom severity of moderate or severe. Future studies with larger samples and inclusion of patients with mild symptom severity are warranted. Moreover, further parameter exploration and safety assessment are needed to provide more convincing evidence of the therapeutic effects of lowintensity tFUS in AD.

In conclusion, our findings suggest that low-intensity tFUS under the threshold for BBB disruption was safely delivered and may have beneficial effects on cerebral glucose metabolism and cognitive function in patients with AD. With its exquisite spatial selectivity and significant depth penetration, tFUS may have the potential to serve as a novel non-invasive brain stimulation tool of both cortical and deep brain areas for the treatment of $A D$. As the field of tFUS-mediated neuromodulation is still in its developmental infancy, further studies are warranted to understand its therapeutic mechanism, safety, and efficacy.

ORCID: Hyeonseok Jeong: https://orcid.org/0000-0002-9245-053X; Jooyeon Jamie Im: https://orcid.org/0000-0002-6890-0019; Jong-Sik Park: https://orcid.org/00000003-0142-7076; Seung-Hee Na: https://orcid.org/0000-0001-8578-8578; Wonhye Lee: https://orcid.org/0000-0002-4203-2209; Seung-Schik Yoo: https://orcid.org/00000002-5150-9857; In-Uk Song: https://orcid.org/0000-0002-0181-0844; Yong-An Chung: https://orcid.org/0000-0003-4004-4019

\section{Author Contributions}

Conceptualization: Song IU, Chung YA, Yoo SS. Data acquisition: Jeong H, Im JJ, Park JS, Na SH, Song IU, Chung YA. Data analysis or interpretation: Jeong $\mathrm{H}, \mathrm{Im} \mathrm{JJ}$, Song IU, Chung YA. Drafting of the manuscript: Jeong $\mathrm{H}, \mathrm{Im} J$ J, Lee W, Yoo SS, Song IU, Chung YA. Critical revision of the manuscript: Jeong $H$, Im JJ, Lee W, Yoo SS, Song IU, Chung YA. Approval of the final version of the manuscript: all authors.

\section{Conflict of Interest}

No potential conflict of interest relevant to this article was reported.

\section{Acknowledgements}

We would like to acknowledge Linda B. Xu and Dr. Kavin Kowsari for their editorial support. Figure 1 was designed using resources from Flaticon.com.

This project was supported by a loan of the image-guided tFUS system, a grant from Neurosona Co., Ltd., the Korea Health Industry Development Institute (KHIDI) and Korea Dementia Research Center (KDRC) (HU21C0081), the National Research Foundation of Korea (NRF) funded by the Korean government (2020R1C1C1007254), and a grant of Translational R\&D Project through Institute for BioMedical convergence, Incheon St. Mary's Hospital, the Catholic University of Korea.

\section{References}

1. Khachaturian ZS. Diagnosis of Alzheimer's disease. Arch Neurol 1985:42:1097-1105.

2. Mathis $C A$, Wang $Y$, Klunk WE. Imaging beta-amyloid plaques and neurofibrillary tangles in the aging human brain. Curr Pharm Des 2004;10:1469-1492.

3. Izadifar Z, Babyn P, Chapman D. Mechanical and biological effects of ultrasound: a review of present knowledge. Ultrasound Med Biol 2017;43:1085-1104.

4. Lipsman N, Meng Y, Bethune AJ, Huang Y, Lam B, Masellis M, et al. Blood-brain barrier opening in Alzheimer's disease using MR- 
guided focused ultrasound. Nat Commun 2018;9:2336.

5. Darrow DP. Focused ultrasound for neuromodulation. Neurotherapeutics 2019;16:88-99.

6. Cammalleri A, Croce P, Lee W, Yoon K, Yoo SS. Therapeutic potentials of localized blood-brain barrier disruption by noninvasive transcranial focused ultrasound: a technical review. J Clin Neurophysiol 2020;37:104-117.

7. Aryal M, Vykhodtseva N, Zhang YZ, McDannold N. Multiple sessions of liposomal doxorubicin delivery via focused ultrasound mediated blood-brain barrier disruption: a safety study. J Control Release 2015;204:60-69.

8. Jordao JF, Ayala-Grosso CA, Markham K, Huang Y, Chopra R, McLaurin J, et al. Antibodies targeted to the brain with imageguided focused ultrasound reduces amyloid-beta plaque load in the TgCRND8 mouse model of Alzheimer's disease. PLoS One 2010;5:e10549.

9. Thevenot E, Jordao JF, O'Reilly MA, Markham K, Weng YQ, Foust $K D$, et al. Targeted delivery of self-complementary adeno-associated virus serotype 9 to the brain, using magnetic resonance imagingguided focused ultrasound. Hum Gene Ther 2012;23:1144-1155.

10. Burgess A, Dubey $S$, Yeung $S$, Hough O, Eterman N, Aubert I, et al. Alzheimer disease in a mouse model: MR imaging-guided focused ultrasound targeted to the hippocampus opens the blood-brain barrier and improves pathologic abnormalities and behavior. Radiology 2014;273:736-745.

11. Scarcelli T, Jordao JF, O'Reilly MA, Ellens N, Hynynen K, Aubert I. Stimulation of hippocampal neurogenesis by transcranial focused ultrasound and microbubbles in adult mice. Brain Stimul 2014;7:304-307.

12. Meng Y, Maclntosh BJ, Shirzadi Z, Kiss A, Bethune A, Heyn C, et al. Resting state functional connectivity changes after MR-guided focused ultrasound mediated blood-brain barrier opening in patients with Alzheimer's disease. Neuroimage 2019;200:275-280.

13. D'Haese PF, Ranjan M, Song A, Haut MW, Carpenter J, Dieb G, et al. Beta-amyloid plaque reduction in the hippocampus after focused ultrasound-induced blood-brain barrier opening in Alzheimer's disease. Front Hum Neurosci 2020;14:593672.

14. American Psychiatric Association. Diagnostic and statistical manual of mental disorders (DSM-IV-TR). Washington, DC: American Psychiatric Association, 2000.

15. McKhann G, Drachman D, Folstein M, Katzman R, Price D, Stadlan
EM. Clinical diagnosis of Alzheimer's disease: report of the NINCDS-ADRDA Work Group under the auspices of Department of Health and Human Services Task Force on Alzheimer's Disease. Neurology 1984;34:939-944.

16. Lee W, Chung YA, Jung Y, Song IU, Yoo SS. Simultaneous acoustic stimulation of human primary and secondary somatosensory cortices using transcranial focused ultrasound. BMC Neurosci 2016;17:68.

17. Lee W, Kim H, Jung Y, Song IU, Chung YA, Yoo SS. Image-guided transcranial focused ultrasound stimulates human primary somatosensory cortex. Sci Rep 2015:5:8743.

18. Maes F, Collignon A, Vandermeulen D, Marchal G, Suetens P. Multimodality image registration by maximization of mutual information. IEEE Trans Med Imaging 1997;16:187-198.

19. Bell AJ, Sejnowski TJ. The "independent components" of natural scenes are edge filters. Vision Res 1997;37:3327-3338.

20. Himberg J, Hyvarinen A, Esposito F. Validating the independent components of neuroimaging time series via clustering and visualization. Neuroimage 2004;22:1214-1222.

21. Yoon $\mathrm{K}$, Lee $\mathrm{W}$, Chen $\mathrm{E}$, Lee JE, Croce $\mathrm{P}$, Cammalleri $\mathrm{A}$, et al. Localized blood-brain barrier opening in ovine model using imageguided transcranial focused ultrasound. Ultrasound Med Bio 2019;45:2391-2404.

22. Hynynen K, McDannold N, Vykhodtseva N, Raymond S, Weissleder $R$, Jolesz FA, et al. Focal disruption of the blood-brain barrier due to $260-\mathrm{kHz}$ ultrasound bursts: a method for molecular imaging and targeted drug delivery. J Neurosurg 2006;105:445-454.

23. Wu SY, Aurup C, Sanchez CS, Grondin J, Zheng W, Kamimura $\mathrm{H}$, et al. Efficient blood-brain barrier opening in primates with neuronavigation-guided ultrasound and real-time acoustic mapping. Sci Rep 2018;8:7978.

24. Varghese T, Sheelakumari R, James JS, Mathuranath P. A review of neuroimaging biomarkers of Alzheimer's disease. Neurol Asia 2013;18:239-248.

25. Ongur $\mathrm{D}$, Price JL. The organization of networks within the orbital and medial prefrontal cortex of rats, monkeys and humans. Cereb Cortex 2000;10:206-219.

26. Godsil BP, Kiss JP, Spedding M, Jay TM. The hippocampalprefrontal pathway: the weak link in psychiatric disorders? Eur Neuropsychopharmacol 2013;23:1165-1181. 